\title{
Existence of Ipsilateral Hemiparesis in Ischemic and Hemorrhagic Stroke: Two Case Reports and Review of the Literature
}

\section{F. Saada N. Antonios}

University of Florida College of Medicine, Jacksonville, Fla., USA

\section{Key Words}

Ischemic stroke $\cdot$ Hemorrhagic stroke $\cdot$ Ipsilateral

hemiparesis

\begin{abstract}
Supratentorial stroke presenting as contralateral hemiparesis is predominantly related to contralateral projection of the corticospinal tract. While most corticospinal fibers decussate at the level of the medulla, some tracts continue descending as ipsilateral ventral corticospinal fibers. In this systematic review of the literature and 2 case reports specifically seen in our institute, we describe the presence of ipsilateral hemiparesis in ischemic and hemorrhagic stroke patients. We compiled available information about the existence of uncrossed corticospinal tracts and/or the presence of bilateral operating motor cortical networks. There was an association in patients with congenital uncrossed corticospinal tracts and posterior fossa malformations. There was also a high correlation of ipsilateral hemiparesis in patients with remote infarction. A previous stroke was seen in $50 \%$ of the patients, all except for 1 case were ischemic in nature. Patients with previous infarcts do demonstrate an adaptive compensation for damaged or disconnected regions of an injured area. This emphasizes the need to consider the investigation for previous infarctions or underlying genetic structural defects by using certain imaging modality.

(c) 2013 S. Karger AG, Basel
\end{abstract}

\section{Introduction}

Supratentorial stroke is commonly recognized as causing neurological impairment of movement on the contralateral side of the body. The reason for this is felt to be the predominance of contralateral corticospinal projections, which arise from the cortical regions of the brain and decussate in the caudal medulla before traveling to the spinal cord [1]. While not all fibers decussate, $70-90 \%$ of them do, resulting in this 'crossed' clinical finding [2]. Prior neuroanatomical studies have firmly supported the notion that the primary motor cortex predominantly innervates the contralateral half of the body [3]. This was demonstrated by Dejerine [4] and Foerster [5], when stereotactic procedures performed for relief of parkinsonism were noted to produce contralateral hemiparesis.

However, as Nyberg-Hansen [6] demonstrated in his studies involving primates, cats, and adult rats, projection of pyramidal tract neurons to the ipsilateral spinal cord does exist. He used the silver impregnation method for microglial staining; by labeling axons with a high resolution axonal tracer, it was found that some aberrant corticospinal axons travel in the ipsilateral dorsal funiculus, most then descend through the contralateral spinal cord, then recross in the midline or near their level of termination [6].

\section{KARGER}

E-Mail karger@karger.com

www.karger.com/ene
(C) 2013 S. Karger AG, Basel

0014-3022/14/0712-0025\$39.50/0
Fahed Saada, MD

University of Florida College of Medicine

$580 \mathrm{~W} 8$ th Street, Tower 1, 7th Floor

Jacksonville, FL 32209 (USA)

E-Mail fahed.saada@jax.ufl.edu 
Humans are particularly interesting because in some individuals, as many as $30 \%$ of the corticospinal axons descend as the ipsilateral ventral corticospinal tract [3]. A careful quantitative motor examination of patients with a contralateral hemiparesis will at times reveal some weakness in the ipsilateral arm and leg. When examiners use the ipsilateral limbs for comparison of motor strength, they notice only that the contralateral limbs are much weaker; they fail to recognize that the ipsilateral limbs are also weak. Noskin et al. [7] investigated this idea further. They examined 30 patients with first-ever unilateral hemiparetic subcortical ischemic stroke by performing hand dynamometry and the 9-hole peg test for the evaluation of grip strength and dexterity, respectively. The researchers found that ipsilateral motor deficits were present immediately after stroke and extended into the subacute and chronic recovery periods. Furthermore, a report by Johansen-Berg et al. [8] indicated that increased activation in ipsilateral cortical motor areas occurs during movements of a paretic hand, as shown by transcranial magnetic stimulation performed on 11 patients after first ischemic left middle cerebral artery territory stroke compared to 16 healthy controls. While this may represent a functionally relevant adaptive response to the associated brain injury in the affected patients, the finding could reflect the relatively increased recruitment of uncrossed corticospinal projections from the motor cortex [8]. Not only lack of crossing of fibers, but also bilateral cortical neuronal networks are sometimes involved in the phenomenon of ipsilateral hemiparesis after stroke, as has been shown in a few well-studied cases $[8,9]$. In these cases, variations in functional significance in determining the degree of neurological deficit were determined using transcranial magnetic stimulation, which interfered transiently with processing in the ipsilateral primary motor or dorsal premotor cortex, and functional magnetic resonance imaging (fMRI) to quantify activation of ipsilateral motor areas during simple and choice reaction time tasks [10].

For neurologists and most other medical professionals, the idea that each hemisphere controls the opposite half of the body, indicating that the nerve fibers of the corticospinal tract cross the midline, does impact their daily practice. Patients and their families are routinely instructed regarding this mechanism, and they readily accept this concept when it is presented to them. However, this is not always true.

The aim of our article is to provide a picture of the present level of understanding of the development of corticospinal projections to the practicing physician and to compile the available information about the existence of uncrossed corticospinal tracts and/or the presence of bilateral operating motor cortical networks. The latter consideration may play a role in patients' recovery status, in those cases in which ipsilateral corticospinal fibers or the contralateral cortex may take over after an injury.

In this article, we present 2 new cases of ipsilateral hemiparesis in patients with ischemic stroke seen in our institution, and also review the available literature regarding all reported cases of ipsilateral weakness from acute unilateral ischemic or hemorrhagic stroke, summarizing and discussing the issue of ipsilateral weakness after disruption of the corticospinal pathway as it relates specifically to these patients. Although there are articles about the measurement of proven ipsilateral motor dysfunction with contralateral weakness, to our knowledge ours is the first comprehensive review of ipsilateral hemiparesis due to ischemic or hemorrhagic stroke.

\section{Materials and Methods}

Published case series and case reports of acute ipsilateral weakness due to either ischemic or hemorrhagic stroke were identified by using the search terms ipsilateral hemiparesis, ipsilateral stroke, and ipsilateral TIA in PubMed and BIOSIS databases. We searched only the English literature; therefore, the possibility of reported cases in the remaining world literature could not be excluded. We were able to identify and include in this search 3 Japanese and 1 Korean case report that had English abstracts. Bibliographies of all articles found by the searches were reviewed to ensure that other published articles meeting the search criteria were not overlooked.

We defined ipsilateral weakness as paresis ipsilateral to the side of the cerebral ischemic or hemorrhagic event with an acute presentation. Patients who had remote infarcts with new neurological weakness were also included. The data gathered included age, gender, type of stroke, lesion location, hemispheric dominance, and number of previous strokes and their locations. We included all of the authors' diagnoses as they were cited in their manuscripts including other pathological findings besides stroke.

\section{Results}

An exhaustive search of ipsilateral hemiparesis, ipsilateral stroke, and ipsilateral TIA yielded 16 publications in PubMed and 10 in BIOSIS. Ipsilateral hemiplegia + stroke yielded no publications in either PubMed or BIOSIS. After excluding nonrelevant articles and including selected relevant references in the identified articles of the search, we ended up with 22 publications that were specifically related to ipsilateral weakness associated with stroke. We found 1 further case by searching references of obtained articles. The nonrelevant articles that were 
Table 1. Summary of case reports and series of ipsilateral hemiparesis/plegia with unilateral stroke

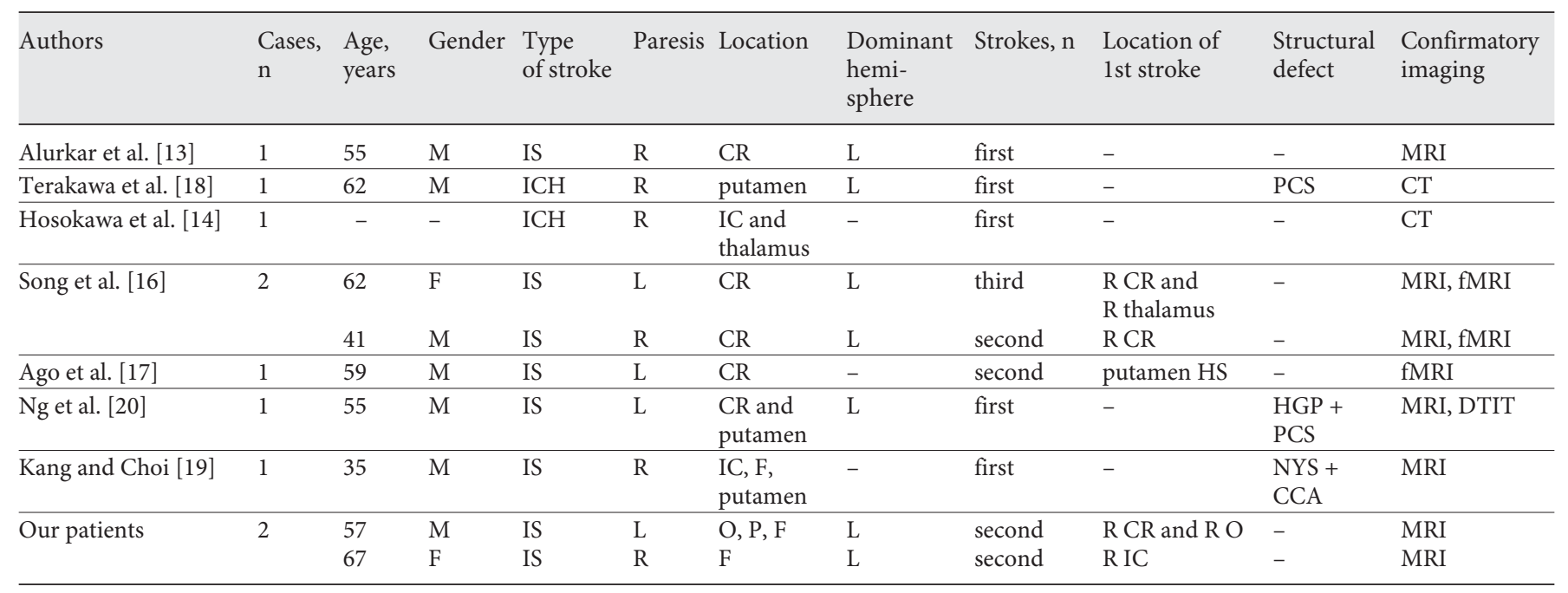

$\mathrm{L}=$ Left; $\mathrm{R}=$ Right; $\mathrm{IS}=$ ischemic stroke; ICH = intracerebral hemorrhage; $\mathrm{IC}=$ internal capsule; $\mathrm{CR}=$ corona radiata; $\mathrm{O}=$ occipital; $\mathrm{P}=$ parietal; $\mathrm{F}=$ frontal; HS = hemorrhagic stroke; HGP = horizontal gaze palsy; PCS = progressive congenital scoliosis; NYS = nystagmus; CCA = corpus callosum agenesis; DTIT = diffusion tensor imaging tractography.

excluded contained 2 cases: Kudo and Uno [11] reported a case of a 62-year-old woman presenting with right hemiparesis and CT findings of subarachnoid hemorrhage in the right cistern of the sylvian fissure; Schneider and Crosby [12] reported the other case in 1983, which was that of a patient with ipsilateral hemiparesthesia secondary to an arteriovenous malformation located in the supplementary sensory area near the island of Reil.

After the above exclusions, we were left with a total of 6 single case reports and 1 report that included 2 cases, for a total of 8 cases from the literature. All of these cases were hospital-based and were reported retrospectively. All of these cases had an ischemic or hemorrhagic stroke and an acute neurological clinical event that the authors ascribed as being related to the ipsilateral weakness. No population-based series were found. After including our 2 cases, we had a total of 10 cases available for analysis.

Table 1 shows the demographics including age, gender, type of stroke, location, side of paresis, dominance, previous strokes, predisposing comorbidity, and imaging modality. A total of 10 patients were included in the table. There were 7 men, 2 women and 1 whose gender was not reported, with age at presentation varying from 35 to 67 years. Ischemic supratentorial stroke was seen in 8 of 10 patients. There were 2 hemorrhagic strokes (intracerebral hemorrhages); one was located in the putamen and the other in the internal capsule and thalamus. Seven patients had left hemisphere dominance, with the remaining 3 unknown.

The strokes were seen in the right nondominant hemisphere in 4 of 10 patients and were seen in the right hemisphere in 2 additional cases in which cerebral dominance was not reported. The exact location of these strokes varied. Five of these patients had subcortical infarctions and one had a cortical (frontal) infarct. The subcortical infarcts were located in the corona radiata (5 cases), the putamen ( 3 cases), and the thalamus ( 1 case). Four patients had multiple areas of the unilateral hemisphere involved (3 subcortical, 1 cortical). Remote infarcts were seen almost in $50 \%$ of the patients, and all of these were ischemic in nature. All previous strokes had caused contralateral hemiparesis. Two of these had same side hemisphere recurrent acute stroke, which this time caused ipsilateral hemiparesis.

Underlying structural disease was seen in 3 cases. Two patients had progressive congenital scoliosis, and one had agenesis of the corpus callosum. Two of the 3 patients had the clinical presentation of ocular symptoms consisting of nystagmus and horizontal gaze palsy with hemiparesis that was unrelated to brainstem pathology.

MRI with diffusion-weighted imaging (DWI) was performed in 7 of 10 cases, all at the time of acute presentation. Four patients underwent fMRI, 3 of whom also had MRI performed. In 1 case, fMRI was the only imaging modality used. Diffusion tensor imaging tractography 

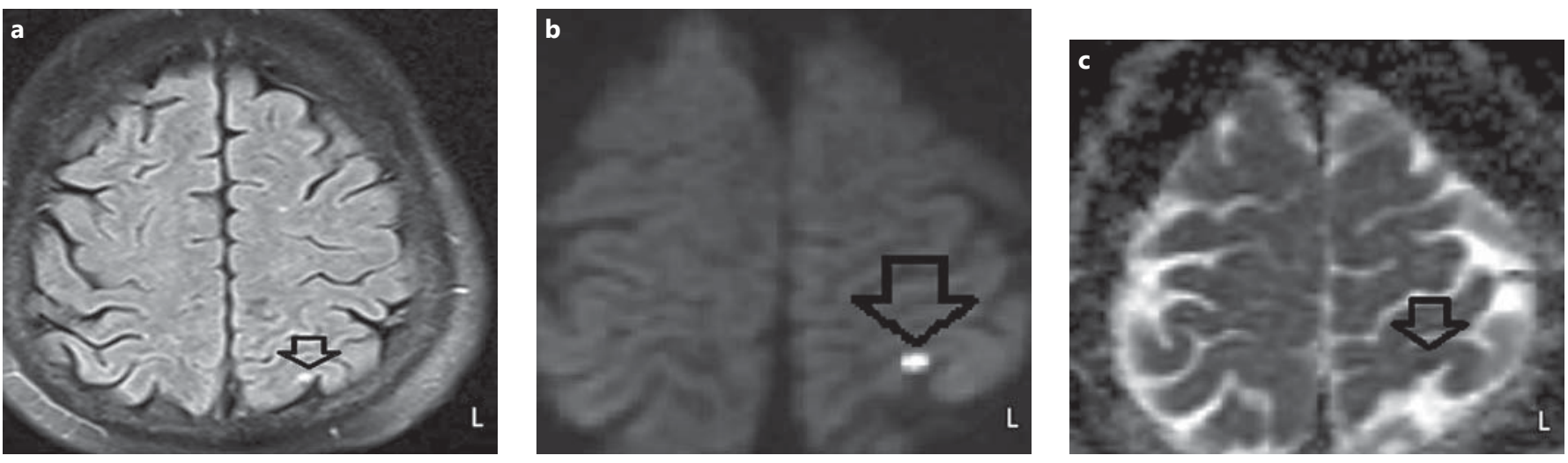

Fig. 1. FLAIR (a) and DWI (b) on the second day of hospital admission revealed a small high-intensity area and punctate areas of restricted diffusion present in the left parietal-superior frontal lobe representing small cortical and white matter infarcts (arrow). The infarcts correlate with apparent diffusion coefficient mapping (c) suggestive of acute infarction (arrow).

was performed in 1 patient with progressive congenital scoliosis, it showed uncrossed corticospinal tracts. CT was performed to diagnose the 2 cases of hemorrhagic stroke (intracerebral hemorrhage).

\section{First Case Report}

A 57-year-old right-handed man with a history of hypertension, dyslipidemia, and tobacco abuse developed sudden onset left hemiparesis and dysarthria. He presented $12 \mathrm{~h}$ after the onset of symptoms. On neurological examination, he had dysarthria, a mild-to-moderate left facial droop, and pronounced left upper extremity weakness graded as 3-4/5 using the MRC grading system. Initial head CT demonstrated small hypodensities near the right basal ganglia region which represented remote infarcts. No acute infarction was apparent. The following day, diffusion MRI of the brain was obtained and showed punctate areas of restricted diffusion in the left parietal-superior frontal lobe representing small acute cortical and white matter infarcts (fig. 1). There was a remote infarct in the right periventricular white matter extending into the right basal ganglia region. A 2-D echocardiogram was unremarkable showing normal left ventricular (LV) size, mild concentric LV hypertrophy, and normal LV function, with an ejection fraction of $60-65 \%$. Telemetry monitoring showed no arrhythmias. Carotid duplex sonography showed an echogenic plaque visualized within the mid aspect of the left common carotid artery with mid and distal left internal carotid artery stenosis. CT angiography was performed and revealed greater than $75 \%$ stenosis of the left internal carotid artery. The patient's weakness persisted throughout the hospital course and after 7 days he underwent a left carotid endarterectomy.

\section{Second Case Report}

A 67-year-old right-handed female with a history of hypertension, diabetes mellitus, and prior right lacunar infarct with residual left hemiparesis presented with a 2-day history of progressively worsening right face, arm and leg weakness. On neurological examination, she had a mild right facial droop, and right hemiparesis graded as $4 / 5$ using the MRC grading system. Initial CT scan of the head showed focal encephalomalacia within the region of the right internal capsule with no acute intracranial hemorrhage, mass effect, or new infarct seen. MRI scan showed a right middle cerebral artery infarction with restricted diffusion over the right frontal operculum (fig. 2). Magnetic resonance angiography was performed and demonstrated loss of flow-related enhancement within the right M1-M2 segments of the right middle cerebral artery. There was also loss of flow-related enhancement in the distal M3 branch with a focus of susceptibility effect likely representing underlying thrombus. Carotid duplex did not detect any significant flow-limiting stenosis according to peak systolic velocity criteria. A 2-D echocardiogram showed severe concentric LV hypertrophy with hyperkinetic left ventricular wall motion and a LV ejection fraction of $75-80 \%$. There were mildly thickened aortic valve leaflets, consistent with aortic sclerosis. The patient's weakness improved but did not completely resolve after 3 days. The patient was discharged to a rehabilitation center.

\section{Discussion}

Ipsilateral hemiparesis after a supratentorial cerebral ischemic or hemorrhagic stroke has rarely been reported. Of note is that when careful neurological examination is performed on patients with acute stroke (whether ischemic or hemorrhagic) involving contralateral hemiparesis, subtle ipsilateral hemiparesis may be found. We describe 10 cases, including 2 of our own, in which strokes ( 8 ischemic, 2 hemorrhagic) caused ipsilateral hemiparesis. Each patient had various processes related to the motor deficit. The most common causes were related to ipsilateral projection from the primary motor cortex [13], 
Fig. 2. a FLAIR signal abnormalities within the subcortical white matter and the $3-\mathrm{mm}$ focus of susceptibility effect projecting over the right frontal operculum (arrow). b DWI demonstrates multiple small areas of restricted diffusion throughout the right middle cerebral artery territory within the basal ganglia and right thalamus (arrows). There is also a lacunar infarct in the right centrum semiovale.
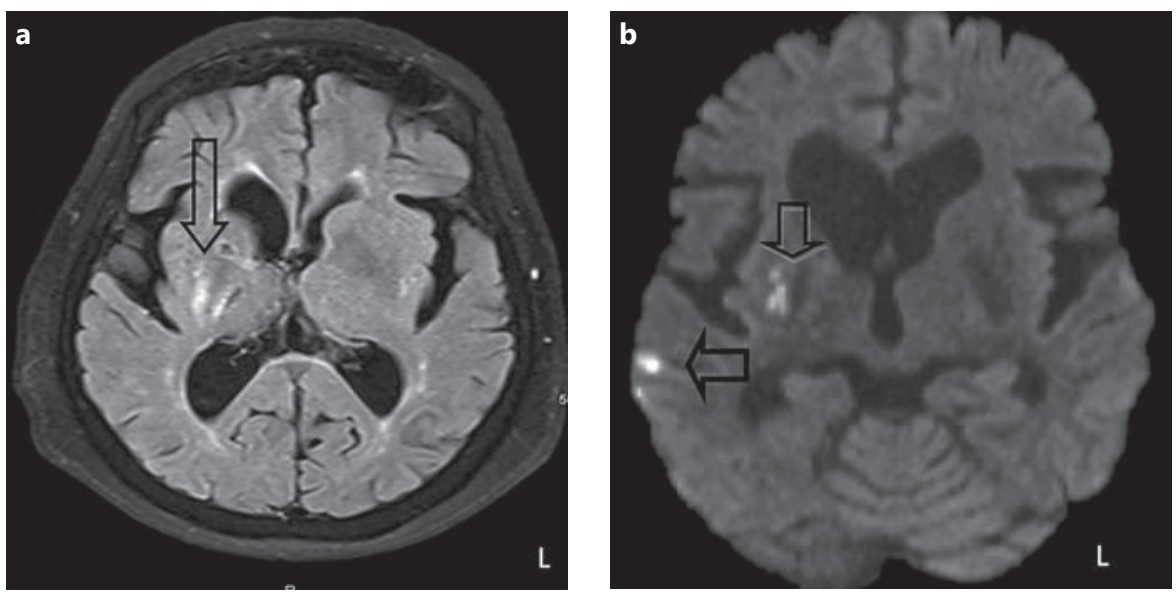

congenitally uncrossed pyramidal tracts [14], lesions that affected the secondary motor area in the precentral insular cortex which bilaterally innervate the face and limbs, or cortical reorganization within the motor areas of the unaffected hemisphere [15].

Our first case was that of a patient with remote asymptomatic contralateral infarcts in imaging studies, who developed acute left hemiparesis associated with ipsilateral (left-sided) parietal-superior frontal lobe cortical and white matter infarcts. Our second case was that of prior contralateral stroke - right lacunar infarct with residual left hemiparesis - who then developed right hemiparesis associated with ipsilateral (right-sided) middle cerebral artery infarction with restricted diffusion over the right frontal operculum. Although we were not able to obtain fMRI or MRI tractography to try to confirm our conclusions, these cases are at least consistent with uncrossed corticospinal tracts. They did not include the primary motor cortex or the secondary precentral insular cortex, although we could not exclude the possibility of cortical reorganization of the unaffected hemisphere since there were old infarcts in both cases.

Song et al. [16] described 2 cases with fMRI confirmation which were similar to the 2 cases we reported from our institution. Song et al.'s patients developed ipsilateral hemiparesis after an ischemic infarction to the left corona radiata. Both of these patients had previously experienced contralateral hemiparesis after right-sided corona radiata ischemic infarcts. In both of Song et al.'s cases, fMRI demonstrated that the left sensorimotor cortex was activated during right-hand movement, whereas the bilateral sensorimotor cortex areas were activated during left-hand movement. The mechanism involved is thought to be re-

Ipsilateral Hemiparesis in Ischemic and Hemorrhagic Stroke lated to functional neuronal reorganization of the ipsilateral hemisphere that develops as a result of a new stroke after a previous stroke on the opposite side. In a similar case of ipsilateral hemiparesis, reported by Ago et al. [17], fMRI demonstrated that gripping with the paretic left hand activated the ipsilateral left motor areas but not the right hemisphere motor areas. This activation pattern differs from that of the cases described by Song et al. [16] since the involvement was of the unilateral rather than bilateral cortex. The difference in activation patterns could have been due to the use of different fMRI protocols or to interindividual variations in brain reorganization. However, even though the activation patterns were different, ipsilateral motor area activation was a common and essential finding.

In our review of the literature, we found that patients who have the congenital anomaly of lack of decussation of the pyramidal tracts have been reported to also have other cerebral malformations. Terakawa et al. [18] described this finding of congenitally uncrossed pyramidal tracts, with ipsilateral projection from the primary motor cortex in a man with a history of congenital scoliosis. The patient developed sudden onset right hemiparesis and was found to have a right internal capsule and basal ganglia hemorrhage on head CT scan. An MRI study showed hemorrhage in the right putamen and another apparent congenital anomaly in the medulla, which was found to have an unusual wedge shape. fMRI was performed during a sequential finger opposition task and showed activation in the motor cortex ipsilateral to the finger movements, but not on the contralateral side. Sensory-evoked potentials showed cortical response ipsilateral to the side of stimulation. The presence of the abnormal shape of the 
medulla in addition to the congenital scoliosis both suggest that congenital anomaly might be responsible for uncrossed pyramidal tracts as well [18].

A similar presentation was seen in a patient described by Kang and Choi [19] with agenesis of the corpus callosum, who developed ipsilateral hemiparesis and spontaneous horizontal nystagmus after an infarct in the right middle cerebral artery territory. MRI revealed the presence of an acute infarct in the right middle and inferior frontal gyri, supramarginal gyrus, insular gyrus, internal capsule, head of caudate nucleus, putamen, and globus pallidus. Transcranial stimulation of the hand area of the cerebral motor cortex produced motor-evoked potentials in the abductor pollicis brevis muscle exclusively on the ipsilateral side. This finding again suggests that anomalies of the decussation of the corticospinal tracts can be found in association with other cerebral anomalies - in this case, agenesis of the corpus callosum.

$\mathrm{Ng}$ et al. [20] described a third case of uncrossed corticospinal tract fibers in association with a congenital anomaly. The case was that of horizontal gaze palsy with progressive scoliosis. This patient had pure left motor stroke as a result of a left corona radiate infarct on DWI. Diffusion tensor imaging tractography confirmed the presence of uncrossed corticospinal tract fibers accounting for the ipsilateral deficit.

Patients with uncrossed pyramidal tracts have also been reported to have other cerebral malformations, such as posterior fossa malformations, occipital encephaloceles, Dandy-Walker malformation, Joubert syndrome, and extensive malformations of the brainstem, such as Mobius syndrome [21, 22].

\section{Conclusion}

Ipsilateral hemiparesis after ischemic or hemorrhagic stroke is a rare finding, but it can develop as a result of a new ischemic or hemorrhagic stroke in patients with or without a previous history of stroke. Our 2 case reports demonstrated ipsilateral hemiparesis associated with new infarct; in 1 case the prior old infarcts were asymptomatic and in the other they were contralateral. Although we were not able to obtain fMRI or MRI tractography to try to confirm our conclusions, these cases are at least consistent with uncrossed corticospinal tracts or cortical reorganization within the unaffected hemisphere. Similar cases to ours have been reported, with one being confirmed with MRI tractography [20]. Patients who have ipsilateral strokes consistent with uncrossed corticospi- nal tracts have often been reported to have other associated cerebral anomalies, such as congenital scoliosis, agenesis of the corpus callosum, wedge-shaped medulla ( 1 case), posterior fossa malformations, occipital encephaloceles, Dandy-Walker malformation, Joubert syndrome, and malformations of the brainstem, such as Mobius syndrome. The existence of uncrossed corticospinal tracts and/or the presence of bilateral operating motor cortical networks, as well as any other possible associated congenital anomalies may be further diagnosed by using certain imaging modalities, like MRI, fMRI, or MRI tractography.

Not all ipsilateral strokes are due to uncrossed corticospinal fibers. Some may be due to cortical reorganization within the motor areas of the unaffected hemisphere. Patients with previous infarcts demonstrate an adaptive compensation for damaged or disconnected regions of the injured area. All of these considerations emphasize the need to consider investigations for previous infarctions, underlying congenital anomalies, or functional reorganization of the cerebral cortex in such patients. In the future, this consideration may play a large role in patients' status for recovery.

\section{Disclosure Statement}

The authors did not receive any funding for this study.

\section{References} spinal projection. Prog Neurobiol 1992;38: 169-202.

2 Nathan P, Smith M, Deacon P: The corticospinal tracts in man. Brain 1990;113:303-324.

> 3 Weiller C, Chollet F, Frinston KJ, Wise RJ, Frackowiak RS: Functional reorganization of the brain in recovery from striatocapsular infarction in man. Ann Neurol 1992;31:463472 .

4 Dejerine J: Anatomi des Centres Nerveux Paris, Rueff El Cie, 1901.

5 Foerster O: Motorische Felder Bahnen, ed 357. Berlin, Handbuch der Neurologie, 1936.

6 Nyberg-Hansen R: Corticospinal fibres from the medial aspect of the cerebral hemisphere in the cat. Exp Brain Res 1969;7:120-132.

$\checkmark 7$ Noskin O, Krakauer JW, Lazar R, Festa J, Handy C, O’Brien K: Ipsilateral motor dysfunction from unilateral stroke: implications for the functional neuroanatomy of hemiparesis. J Neurol Neurosurg Psychiatry 2008;79: 401-406. 
$>$ Johansen-Berg H, Rushworth M, Bogdanovic M, Kischka U, Wimalaratna S, Matthews P: The role of ipsilateral premotor cortex in hand movement after stroke. PNAS 2002;99: 14518-14523.

$>9$ Leonardo R, Cohen G, Hallette M: Role of the ipsilateral motor cortex in voluntary movement. Can J Neurol Sci 1997;24:284-291.

$\checkmark 10$ Eyre A, Phil D, Taylor B, Villagra F, Smith M, Miller S: Evidence of activity-dependent withdrawal of corticospinal projections during human development. Neurology 2001;57: 1543-1554.

11 Kudo T, Uno T: Ipsilateral hemiparesis caused by subarachnoid hemorrhage in a patient with a ruptured middle cerebral arterial aneurysm. Neurosurgery 1984;15:727-729.

-12 Schneider R, Crosby E: Ipsilateral symptoms caused by an arteriovenous malformation of the second or supplementary sensory area of the island of Reil. Neurosurgery 1983;12:557560
13 Alurkar A, Karanam L, Atre P, Nirhale A, Nayak S, Oak S: Ipsilateral stroke with uncrossed pyramidal tracts and underlying right internal carotid artery stenosis treated with percutaneous transluminal angioplasty and stenting: a rare case report and review of the literature. Neuroradiol J 2012;25:237-242.

14 Hosokawa S, Tsuji S, Uozumi T, Matsunaga K, Ota S: Ipsilateral hemiplegia caused by right internal capsule and thalamic hemorrhage. Neurology 1996;46:1146-1149.

15 Weiller C, Chollet F, Friston K: Functional reorganization of the brain in recovery from striatocapsular infarction in man. Ann Neurol 1992;31:463-472.

16 Song YM, Lee Y, Park JM, Yoon BY, Roh JK Ipsilateral hemiparesis caused by a corona radiata infarct after a previous stroke on the opposite side. Arch Neurol 2005;62:809-811.

17 Ago T, Kitazono T, Ooboshi H: Deterioration of pre-existing hemiparesis brought about by subsequent ipsilateral lacunar infarction. J Neurol Neurosurg Psychiatry 2003;74:11521153.
8 Terakawa H, Abe K, Nakamura M, Okazaki T, Obashi J, Yanagihara T: Ipsilateral hemiparesis after putaminal hemorrhage due to uncrossed pyramidal tract. Neurology 2000;54: 1801-1805.

19 Kang K, Choi NC: Ipsilateral hemiparesis and spontaneous horizontal nystagmus caused by middle cerebral artery territory infarct in a patient with agenesis of the corpus callosum. Neurol Sci 2012;33:1165-1168.

20 Ng A, Sitoh YY, Zhao Y, Teng E, Tan L: Ipsilateral stroke in a patient with horizontal gaze palsy with progressive scoliosis and a subcortical infarct. Stroke 2011;42:E1-E3.

21 Lagger RL: Failure of pyramidal tract decussation in the Dandy-Walker syndrome. Report of two cases. J Neurosurg 1979;50:383387.

22 Donkelaar HJ, Lammens M, Wesseling P, Hori A, Keyser A, Rotteveel J: Development and malformations of the human pyramidal tract. J Neurol 2004;251:1429-1442. 